\title{
Cement Pavement Surface Crack Detection Based on Image Processing
}

\author{
Hongwei Lei a, Jianlian Cheng ${ }^{b, *}$, Qi Xu ${ }^{c}$
}

Highway Maintenance Equipment National Engineering Laboratory, Key Laboratory of Road Construction Technology and Equipment Ministry of Education, School of Construction Machinery,Chang'an University,Xi'an 710064,China

*Corresponding Author: Jianlian Cheng, cjlian@chd.edu.cn

Abstract:

This article introduces the application of image recognition technology in cement pavement crack detection and put forward to method for determining threshold about grayscale stretching. This algorithm is designed for binarization which has a self-adaptive characteristic. After the image is preprocessed, we apply 2D wavelet and Laplace operator to process the image. According to the characteristic of pixel of gray image, an algorithm designed on binarization for Binary image. The feasibility of this method can be verified the image processed by comparing with the results of three algorithms: Otsu method, iteration method and fixed threshold method.

Keywords: Pavement crack detection; Grayscale stretching; Self-adaptive; 2D Wavelet; Laplace; Binarization

Citation: H.W. Lei, J.L. Cheng, Q. Xu, Cement Pavement Surface Crack Detection Based on Image Processing. Mech Eng Sci, $2019,1(1)$ : 48-53. https://doi.org/10.33142/me.v1i1.661

\section{Introduction}

Pavement Surface Crack is one of the main damage forms of road. Manual crack detection is time-consuming, laborious and costly. This method is no longer suitable for the market. Road surface image capture and processing techniques are now widely used. However, due to various reasons of interference, the captured image is not easy to identify ${ }^{[1]}$.

In order to solve the problems we encountered, many scholars have done the corresponding research. Fan Jiu-lun ${ }^{[2]}$ proposed the two-dimensional otsu's curve thresholding segmentation method for gray-level images; Lakhwinder Kaur ${ }^{[3]}$ proposed image de-noising NormalShrink threshold method, but it can lead to blurry edges on the target. DONG Hong-Yan ${ }^{[4]}$ proposed the multiscale edge detection based on laplacian pyramid method, this approach enhances the boundary between the target and background; Nobuyuki Otsu ${ }^{[5-9]}$ proposed the Otsu method for binary image conversion, however, when it is applied to pavement crack image processing, the results are not ideal.

In this article, we begin with gray scale transformation, and then we use weighted average method to transform the captured image. Using the improved grayscale stretching algorithm to stretch the gray image. After that, we use Laplace operator to sharpen the abrupt region of the image pixel value. Next step is image de-noising which use $2 \mathrm{D}$ wavelet de-noising method. Finally, an algorithm about binarization that have a self-adaptive characteristic is used to transform the image. The transformed image is compared with the processing results of Otsu method, iteration method and fixed threshold method.

The results show that the grayscale stretching algorithm and line self-adaptive threshold binarization algorithm in this paper presented are effective in image processing.

\section{Image preprocessing}

Images which are captured at high speed usually have three disturbing factors: uneven illumination, random noise and the occlusion of irregular objects. Cement pavement cracks are mainly divided into three types: transverse, longitudinal and irregular oblique cracks. Usually, the color of the crack is dark, that is, the gray-level of the target is low. To sum up, in image processing, it is necessary to enhance the target surface crack and reduce the interference from the background ${ }^{[10]}$.

\subsection{Gray-level transformation}

There are three methods that maximum method, average method and weighted average method for image to transformation its gray-level $^{[11]}$. The maximum method is to select the maximum value from the $R, G$ and $B$ values of the image as the $R, G$ and $B$ values of the new image. That is, as the following formula (1) is shown.

$$
R=G=B=\max (R, G, B)
$$


The gray image obtained by this method is not suitable for pavement crack image processing because of its high brightness. The average method converts the $R, G$ and $B$ values of the image into the average values of the original $R, G$ and $B$ values, as formula (2) shown below:

$R=G=B=\left(\frac{R+G+B}{3}\right)$

The gray image generated by this method is softer than those obtained by other methods, while the processing of cracked image requires enhancing the edge of mutant pixel, so this method is not applicable, too.

The weighted average method is to carry out the weighted average of the values of $\mathrm{R}, \mathrm{G}$ and $\mathrm{B}$ according to a certain weight value, as formula (3) shown below.

$R=G=B=\frac{\left(\omega_{R} R+\omega_{G} G+\omega_{B} B\right)}{3}$

$\omega_{R} 、 \omega_{G} 、 \omega_{B}$ are the weights of $\mathrm{R}, \mathrm{G}$ and $\mathrm{B}$, and different values are taken to form different grayscale images. According to experience, taking $\omega_{G}>\omega_{R}>\omega_{B}$ could make the obtained images easier to recognize. In general, we take $\omega_{R}=0.2989$, $\omega_{B}=0.5870, \omega_{G}=0.1140$, that could obtain better grayscale images at this time, as shown in figure1.

\subsection{Grayscale stretching method}

\subsubsection{Algorithm}

Image quality is often affected by many reasons, which hinders the detection of cracks. Grayscale stretching is a common method to improve image quality.

The histogram of cement pavement crack image is mainly of single-peak type, and the crack of pavement is in the dark part with lower gray-level. The background focuses near the peak. Grayscale stretching is completed through grayscale transformation ${ }^{[12]}$.

Let $l(n, m)$ be the input image, its minimum gray-level $J$ and maximum gray-level $K$ are defined as follows:

$J=\min [l(n, m)]$;

$K=\max [l(n, m)]$

Map $J$ and $K$ linearly to 0 and 255, respectively, and the resulting image $q(n, m)$ is as follows:

$q(n, m)=\left(\frac{255}{J-K}\right)[l(n, m)-J]$

According to the characteristics of road surface image, using stretching method to improve the above formula. The main way of segmental stretching is to divide the gray histogram of pavement crack image into multiple sections, and then conduct linear processing to enhance the recognition degree of the image without losing the original information of the image. The piecewise linear transformation can highlight the grayscale details of the target and suppress the background of the image according to the actual needs ${ }^{[13]}$. The formula (5) is as follows:

$$
q(n, m)= \begin{cases}\frac{m_{1}}{n_{1}} l(n, m), & l(n, m)<n_{1} \\ \frac{m_{2}-m_{1}}{n_{2}-n_{1}}\left[l(n, m)-n_{2}\right]+m_{1}, & n_{1}<l(n, m)<n_{2} \\ \frac{255-m_{2}}{255-n_{2}}\left[l(n, m)-n_{2}\right]+m_{2}, & n_{2}<l(n, m)<255\end{cases}
$$

The grayscale range of the image is $[0,255]$. According to the requirements, the range $n$ of the piecewise function and the slope $m$ of each function are selected, so we divide the gray level of the image into $\left[0, n_{1}\right],\left[n_{1}, n_{2}\right]$ and $\left[n_{2}, 255\right]$, and the second section is stretched, which can effectively suppress the background area and enhance the crack pixel of the road image. However, the numerical selection of $n_{1}$ and $n_{2}$ is a difficult work. According to the characteristics of pixel distribution of different images, different segmentation points can be selected. If the selected segmentation point is not reasonable, it may cause image distortion or enhance the image noise.

\subsubsection{Piecewise function method}

According to the characteristics of cement pavement images, designing the algorithm to determine the threshold values $n_{1}$ and $n_{2}$, The formula (6),(7) are as follows:

$n_{1}=\frac{\sum_{k} x_{i_{1} j_{1}}}{k} \quad\left(i_{1}, j_{1} \in M\right)$

$n_{2}=\frac{\sum_{k} x_{i_{2} j_{2}}}{k} \quad\left(i_{2}, j_{2} \in M\right)$

$n_{1}$ in the piecewise function is the expected value of the last $30 \%$ in the order from the largest to the smallest of the grayscale pixel values. $n_{2}$ in the piecewise function is the expected value of the first $30 \%$ in the order from large to small of the grayscale pixel values. $K$ is $30 \%$ of the total number of pixels. That is, $x_{i_{1} j_{1}}$ and $x_{i_{2} j_{2}}$ are the pixel value.

Grayscale stretching was performed on the crack image, and the results were shown in figure 1. (a), (b) and (c) refer to the original, grayscale and grayscale stretching results.

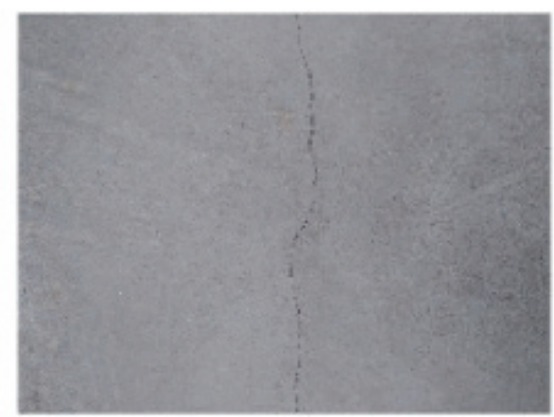

(a) 


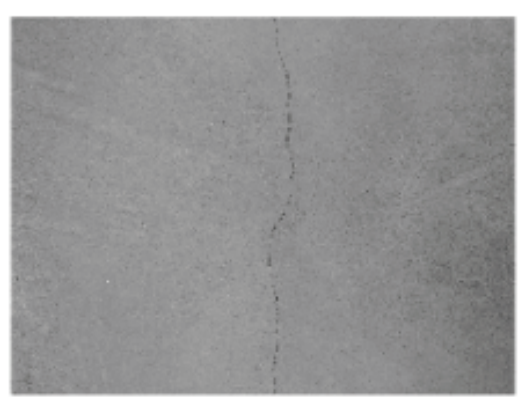

(b)

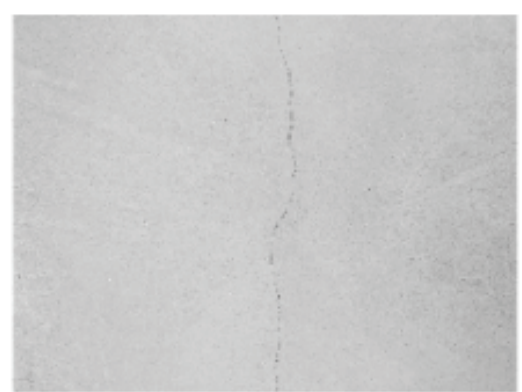

(c)

Figure 1. Image processing results comparison

\section{De-noising method based on 2D wavelet}

Road surface images contain noise, which will cause interference to image grayscale stretching, sharpening and segmentation in the later stage. Due to the variable nature of its window, wavelet transform can maintain the image detail information at the same time of de-noising, that is, narrow window is used when the signal is high frequency, and wide window is used when the signal is low frequency ${ }^{[14]}$.

Denotes the mother wavelet is $\psi(t)$, the scaling factor is $a$ , and the translation factor is $b$, then the wavelet generated by the mother wavelet is :

$\psi_{a, b}(t)=\frac{1}{\sqrt{|a|}} \psi\left(\frac{t-b}{a}\right)$

There we have, $a, b \in R, a \neq 0$.

For the mother wavelet $\psi(t)$, there we have:

$C_{\psi}=\int_{-\infty}^{+\infty} \frac{|\hat{\psi}(\omega)|^{2}}{|\omega|} \mathrm{d} \omega<\infty$

The continuous wavelet transform of signal $f(t)$ is

$w_{f}(a, b)=\left\langle f, \psi_{(a, b)}\right\rangle=|a|^{-\frac{1}{2}} \int_{-\infty}^{+\infty} f(t) \overline{\psi\left(\frac{t-b}{a}\right)} \mathrm{d} t$

$w_{f}(a, b)$ corresponds to the decomposition of $f(t)$ in family of function $\psi_{a, b}(t)$. It can also be regarded as the filtering operation of the original signal $f(t)$ and a set of wavelet bandpass filters of different scales, so that the signal can be decomposed into a series of frequency bands for analysis and processing.

Sample $a$ and $b$, take $a=a_{0}^{m}, b=n b_{0} a_{0}^{m}$, and obtain the discrete wavelet transform (DWT). Generally, $a_{0}=2, b_{0}=1$ are selected. In this case, DWT is called multi-resolution analysis.

Mallat puts forward the tower-type algorithm for solving wavelet coefficients ${ }^{[15]}$, which enables discrete wavelet transform to appear in the form of digital QMF filter Banks. The wavelet decomposition formula of multiresolution analysis is

$$
\left\{\begin{array}{l}
c_{k}^{j-1}=\sum_{n} a_{n-2 k} c_{n}^{j} \\
d_{k}^{j-1}=\sum_{n} b_{n-2 k} c_{n}^{j}
\end{array}\right.
$$

The reconstruction formula is

$c_{k}^{j}=\sum_{n}\left(p_{k-2 n} c_{n}^{j-1}+q_{k-2 n} d_{n}^{j-1}\right)$

Each row and column pixels of the image are processed by Mallat algorithm respectively to complete a $2 \mathrm{D}$ wavelet transform. There we get four subgraphs representing different frequency characteristics and direction characteristics of the original image.

The wavelet transform threshold de-noising method proposed by Donoho et al. ${ }^{[16]}$ is as follows:

Step1: The wavelet coefficients of signals are obtained after wavelet transform of signals.

Step2: The nonlinear threshold $t$ acting on the wavelet coefficient, and obtain the modified coefficient value, That is:

hard threshold method

$\hat{x}=T_{k}(X, t)=\left\{\begin{array}{l}X,|X| \geq t \\ 0,|X|<t\end{array}\right.$

soft threshold method

$\hat{x}=T_{k}(X, t)=\left\{\begin{array}{l}\operatorname{sgn}(X)(|X|-t),|X| \geq t \\ 0,|X|<t\end{array}\right.$

The nonlinear soft threshold is :

$t=\sigma \sqrt{2 \log n}$

Where, $n$ is the signal length, $\sigma$ is the signal standard variance, and $t$ is the estimated threshold level.

Step3: carry out wavelet inverse transformation and reconstruct the signal.

The above $2 \mathrm{D}$ wavelet de-noising method is used to process the original image, and the processed results are shown in figure 2.

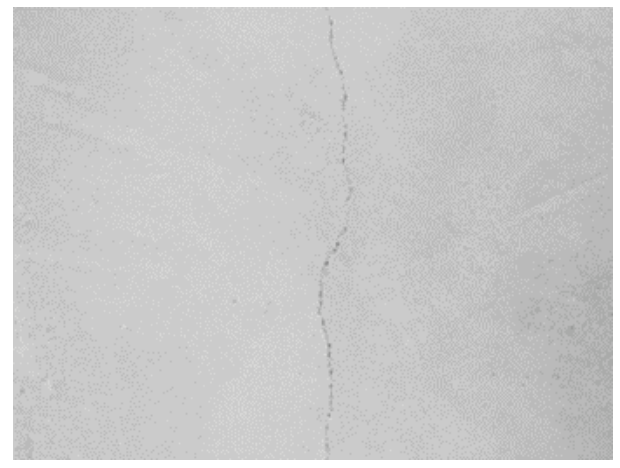

Figure 2. The result of $2 \mathrm{D}$ wavelet de-noising 


\section{Laplace operator transformation}

The main method of image sharpening enhancement is to strengthen the part of image pixel where the value change is bigger, so as to make the contour of image clearer. In other words, when the pixel gray value of the selected center of the neighborhood is lower than the average gray value of other pixels in the field, the gray value of this center pixel will be reduced, and vice versa. In order to achieve image sharpening processing.

Laplace operator is a widely used sharpening operator in image sharpening processing ${ }^{[4]}$.Laplace operator is a kind of second-order differential operator, whose input image will not affect the operator after rotating at a certain angle. For 2D image $f(x, y)$, the general equation of Laplace operator is:

$\nabla^{2} f=\frac{\partial^{2} f}{\partial x^{2}}+\frac{\partial^{2} f}{\partial y^{2}}$

Among them:

$\frac{\partial^{2} f}{\partial x^{2}}=f[x+1, y]-2 f[x, y]+f[x-1, y]$

$\frac{\partial^{2} f}{\partial y^{2}}=f[x, y+1]-2 f[x, y]+f[x, y-1]$

The equation in discrete form is:

$$
\begin{aligned}
\partial^{2} f(x, y)= & f(x+1, y)+f(x-1, y)+ \\
& f(x, y+1)+f(x, y-1)-4 f(x, y)
\end{aligned}
$$

Laplace operator and appropriate filter template are used to sharpen the crack image after gray stretch, and the results are shown in figure 3.

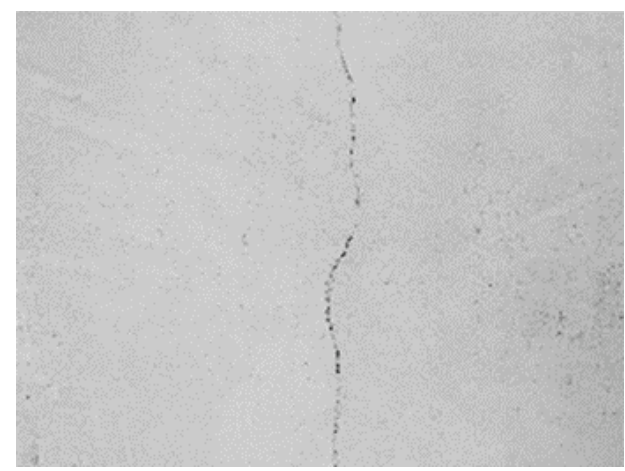

Figure 3. The result of Laplace operator transformation

\section{Methods in this article}

The histogram of the crack image processed by the above method is unimodal. The Ostu method ${ }^{[17]}$ is for obtaining the threshold value of images with bimodal gray histogram. Obviously, it has poor performance in pavement crack image processing. The iterative method retains too much noise when it is applied to pavement crack image processing. The fixed threshold method loses too much information in the work.

R. j. Wall put forward dynamic calculation of the threshold algorithm ${ }^{[18]}$, that divide image into many small pieces, get thresholds from each small piece. In comparison, the perfor- mance of this algorithm is improved. Due to the cracks are line shape, after the processing image in Wall's algorithm kept too much noise, the effect is not ideal.

In view of this situation, this article designs a binarization algorithm for cement pavement crack images. The pixel of cement pavement crack image is mainly divided into two parts: background and target. The pixel value of the target part is obviously lower than that of the background part. Therefore, the common image segmentation algorithm has a large degree of distortion in the image segmentation of cement pavement. It cause the background and the noise get enhancement or the crack target information to lose.

Most of the already proposed adaptive threshold binarization algorithm have been used to segment pixels into chunks, that is, to consider the pixel value characteristics of a local $2 \mathrm{D}$ neighborhood, which leads to inadequate selection of threshold and unsatisfactory processing effect in crack image processing.

According to the characteristics of cement pavement image, designing the adaptive threshold binarization algorithm as follows. The processing image size is $1024 \times 768$.

The operation process of this algorithm is shown in figure 4.

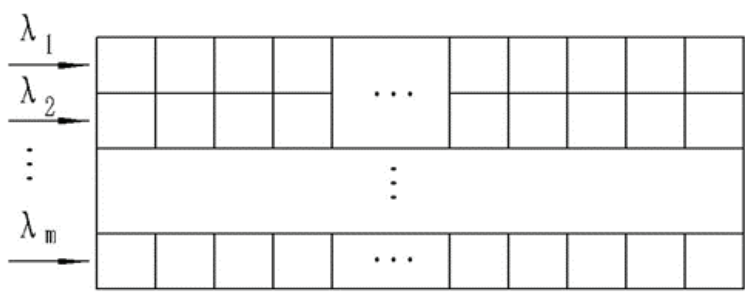

Figure 4. The process of running the line threshold algorithm

Identify the grayscale image as $f(x, y)$, Where, $x \in(1,2, \cdots, m)$ and $y \in(1,2, \cdots, n)$ are the matrix whose pixel of gray image is $m$ rows and $n$ columns, and the element is $a_{x y}$. Each $a_{x y}$ value pair should have a pixel gray value of $a_{x y} \in(0,255)$ . Arrange each row pixel according to its gray value from small to large, then take the first $S$, where $s=\left[\frac{n}{10 \times t}\right], t \in R$.

After many experiments, when the pavement crack image select $t=0.98$ processing effect is good. Then the threshold of line $x$ is $\lambda_{x}=\frac{a_{x 1}+a_{x 2}+a_{x 3}+\cdots+a_{x s}}{s}$, and the pixel whose gray value is bigger than $\lambda_{x}$, then set it equal to 255 ; If pixels with a grayscale value less than $\lambda_{x}$, let set it equal to 0 .

Traverse all the pixels of the image, that is, complete the binarization transformation of the gray image.

\section{Conclusions}

The pavement crack images processed by gray stretch, Laplace sharp and 2D wavelet threshold de-noising were converted into binary-valued images respectively by Ostu method, iterative method and fixed threshold method and the method desighed in this article. The results are shown in figure 5, where (a), (b), (c) and (d) are the processing results of Ostu method, iteration method, fixed value method and line threshold algorithm. 


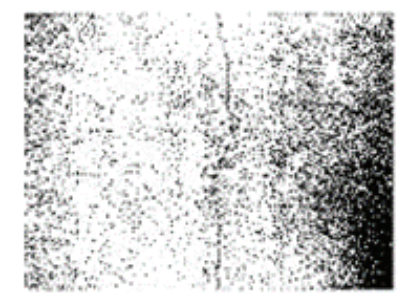

(a)

(b)

(c)

Figure 5. Comparison of binarization results

In figure 6, figure 7, figure 8 and figure 9 are project charts of processing results of Ostu method, iteration method, fixed value method and line threshold algorithm. It can be seen that the noise in the image vertical projection processed by Ostu method seriously affects the recognition of crack location; The image processed by iterative method is lost information and the image has large noise interference. After the fixed threshold method, the target crack information is lost seriously. Line threshold algorithm, the threshold binarization algorithm proposed in this paper, lost fewer information after processing.

Computer technology improves the efficiency of maintaining the pavement. It saves labor cost and time cost. Compared with other binarization methods, the method proposed in this paper is more effective in image processing of pavement cracks. It loses less information and the resulting image is more recognizable.
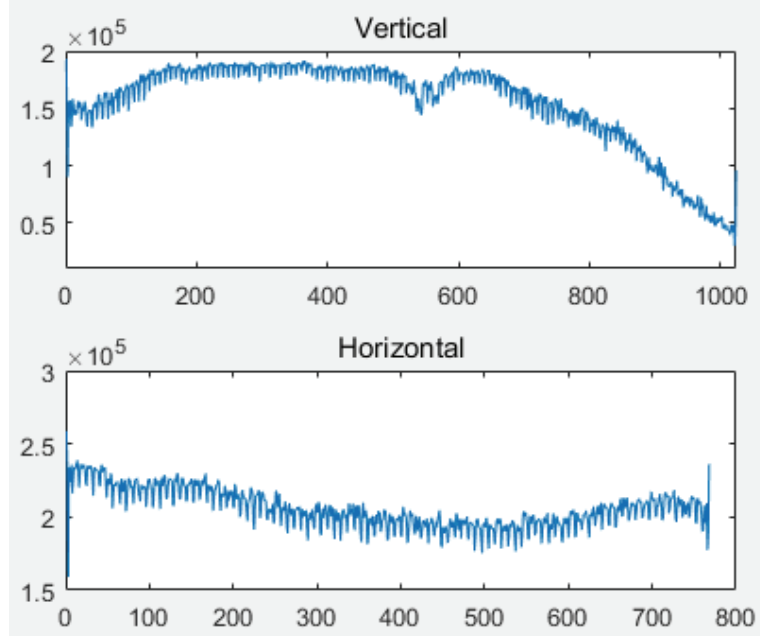

Figure 6. The projection of Ostu method processing results
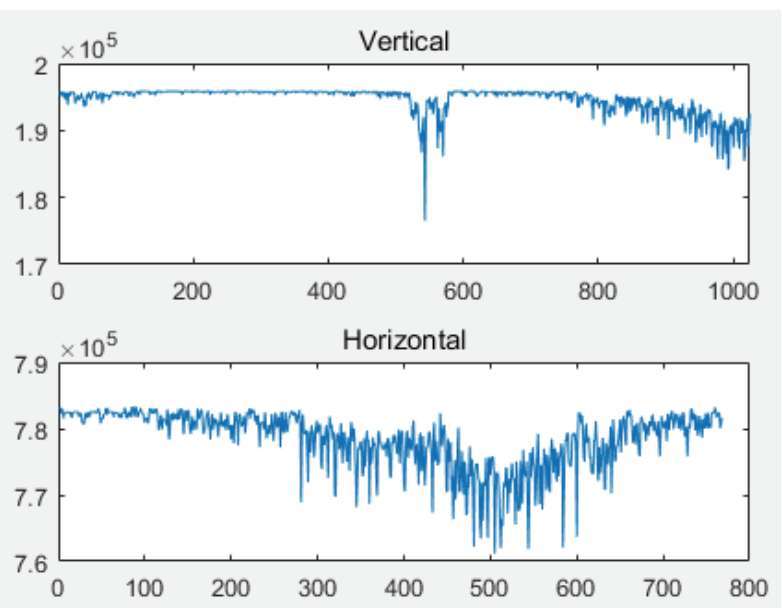

Figure 7. The projection of iteration method processing
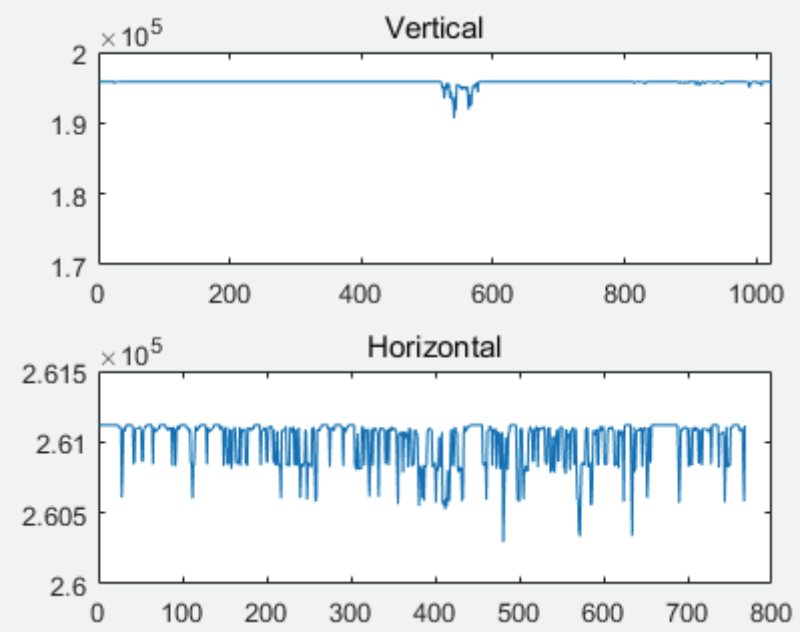

Figure 8. The projection of fixed value method processing result
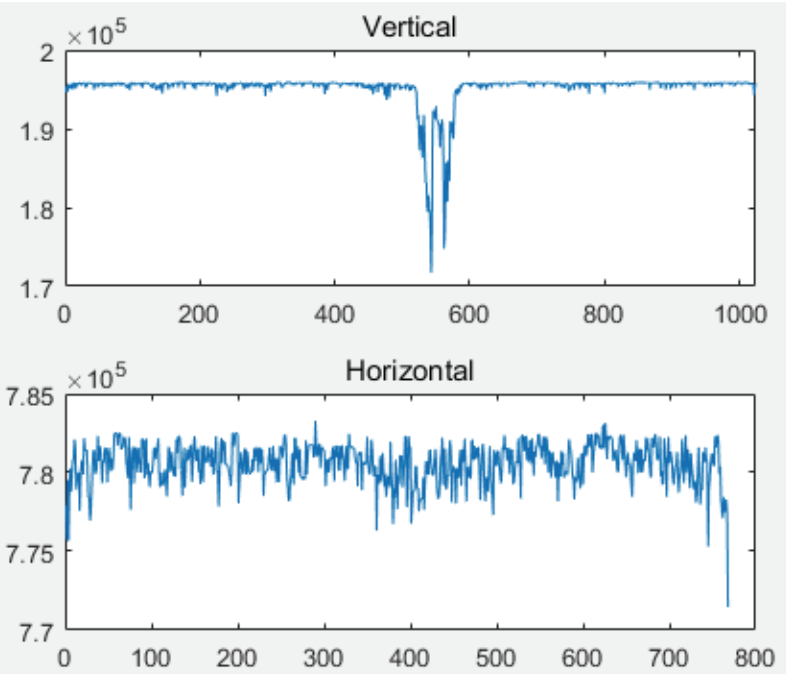

Figure 9. The projection of line threshold method processing result

\section{References}

[1] CHUO Er-yong, Development Summary of International Pavement Surface Distress Automatic Survey System [J]. Transport Standardization, 2009, 09: 96-97;

[2] FAN Jiu-lun, ZHAO Feng, Two-Dimensional Otsu's Curve 
Thresholding Segmentation Method for Gray-Level Images [J], Acta Electronica Sinica,2007,35(4):751-755;

[3] Lakhwinder KAUR, Savita GUPTA, R.C. CHAUHAN. Image Denoising using Wavelet Thresholding [A]. Proceedings of the Third Indian Conference on Computer Vision, Graphics \& Image Processing [C]. Ahmadabad, India: Allied Publishers Private Limited, 2002, 11:1522-1531;

[4] DONG Hong-yan, WANG Lei, LI Ji-cheng, SHEN Zhen-kang, Multiscale edge detection based on Laplacian pyramid [J], Opto-Electronic Engineering,2007,7(34):135-140;

[5] Ohtsu N.A threshold selection method from gray-level histograms [J].IEEE Transactions on Systems Man \& Cybernetics, 2007, 9 (1) : 62-66.

[6] Ferman A M, Tekalp A M, Mehrotra R.Robust color histogram descriptors for video segment retrieval and identification [J].IEEE Transactions on Image Processing;A Publication of the IEEE Signal Processing Society, 2002, 11(5) : 497-508;

[7] Zhang C, Xie Y, Liu D, et al.Fast threshold image segmentation based on 2D fuzzy fisher and random local optimized QPSO [J].IEEE Transactions on Image Processing, 2017, 26(3): 1355-1362;

[8] Qi Lin, Wang Jing, Chen Yanlei, et al. Research on the image segmentation of icing line based on NSCT and 2-D OSTU[C], International Conference on Modelling, Identification and Control, 2015: 1-5;

[9] Liu S.Image segmentation technology of the Ostu method for image materials based on binary PSO algorithm[M],
Advances in computer science, intelligent system and environment. Berlin Heidelberg: Springer, 2011 : 415-419;

[10] PENG Bo1, JIANG Yang-sheng, HAN Shi-fan1, LUO Nanxin, A Review of Automatic Pavement Crack Image Recognition Algorithms[J], Journal of Highway and Transportation Research and Development, 2014, 7, 7(31): 10-25;

[11] Gu Mehua, Su Binbin, Wang Miaomiao, Wang Zhilei, Survey on decolorization methods[J], Application Research of Computers, 2018, 36(5):

[12] Hideyuki Tamura, Computer image processing technology $[\mathrm{M}]$ 。Beijing Normal University Publishing House, 1986 ;

[13] Rafael C. Gonzalez, Richard E. Woods, Digital image processing $[\mathrm{M}]$, Publishing House of Electronics Industry, 2017;

[14] 马拉特, A wavelet tour of signal processing[M].China Machine Press, 2003

[15] Angel P, Morris C, Analyzing the Mallat wavelet transform to delineate contour and textural features $[\mathrm{J}]$. Computer Vision and Image Understanding, 2000, 80 (3) : 267-288;

[16] D.L.Donoho, I.M.JohnStone.Ideal spatial adaptation via wavelet shrinkage[J]. Biometrika, 1994, 81: 425-455;

[17] Otsu N.A threshold selection method from gray-level histo-grams[J].IEEE Transactions on Systems,Man and Cyber-netics, 1979,9(1):62-66

[18] Castleman, K. Digital Image Processing. Prentice-Hall Signal Pro-cessing Series, 1979. 